Military Technical College Kobry El-Kobbah, Cairo, Egypt

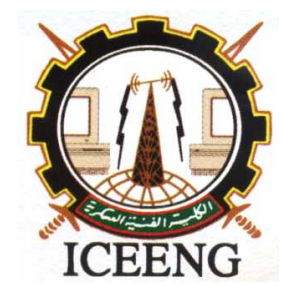

\author{
$6^{\text {th }}$ International Conference \\ on Electrical Engineering \\ ICEENG 2008
}

\title{
A Fuzzy Decision Tree Classifier for Recognition of Fuzzy Image Regions
}

By
M. Zaki*
M. Y. El Nahas *
Eraky Atta **

\section{Abstract:}

In this paper we present a framework for image segmentation and fuzzy region recognition based on fuzzy decision tree (FDT) classifier. The inference of the fuzzy decision tree starts by construction of crisp classification and regression tree (CART), then fuzzification of decision boundary at each node. Fuzzy regions in digital images are extracted by fuzzy c- means algorithm. Then fuzzy regions are matched with fuzzy tree models constructed for sample images from the application domain. This procedure was applied on MRI sample images for identification of normal and abnormal tissues. It was also applied for diagnosis of pathological brain cases by identification of shape and tissue of fuzzy regions. Experimental results show low error rate for the cases under study. Comparison of error rates obtained by this FDT classifier and the CART classifier shows a slight difference of $0.1 \%$ between the two classifiers.

\section{Keywords:}

Fuzzy decision tree, fuzzy clustering, image segmentation, MRI images

* College of Engineering, Al-Azhar University, Cairo, Egypt

** Egyptian Armed Forces 


\section{Introduction:}

In this paper we present a framework for image segmentation into fuzzy regions and their subsequent recognition using a fuzzy decision tree classifier. The fuzzy decision tree classifier is used for automatic segmentation of input images and identification of predefined patterns. The motivation of this research work is the increasing number of applications of automatic image segmentation and shape classification under high degree of uncertainty [9], [12] and [18].

Decision trees are widely used in pattern recognition and artificial intelligence applications. Their popularities are due to their robust performance as sequential nonparametric classifiers. A decision tree can be viewed as a set of decision rules which can be easily interpreted by humans. The most popular derision tree algorithms are the classification and regression tree (CART), third interactive dichotomizer (ID3) and its successor C4.5 [8]. These decision trees can be named crisp decision trees since the decision at each node of the tree is binary decision. While this is favorable for rapid classification of patterns, it is not necessary the best choice when patterns classes reveal great uncertainty. For this reason fuzzy decision tree has been proposed [13] to allow gradual transition to exist between features values at decision nodes of the tree. Additional strength of fuzzy decision trees compared to other methods of recognition is the ease with which they can be extended to nonnumeric domains. This allows fuzzy decision trees to be used when patterns features are fuzzy. The inference of fuzzy decision tree and its performance evaluation has been studied by [6], [13] and [19].

In applications domains of shape recognition, frequently the region's shape and boundary reveal great uncertainty which renders the learning and recognition of these image structures sometimes difficult. The use of dynamic trees for image segmentation and recognition based on statistical model has been suggested for these applications [20]. Image recognition by splitting images into trees of fuzzy regions has also been proposed by [22] but there approach is limited by extraction of topological regions.

Medical image applications are one of these domains where fuzzy shape description should be efficient. In medical diagnosis based on magnetic resonance imaging (MRI) techniques, the segmentation of brain images and the identification of pathological cases from their shapes or tissues are routine tasks conducted by highly experienced specialists. This makes it costly and time consuming procedure. Automatic segmentation of brain tissues by fuzzy c-means algorithm has been proposed [5]. Most of the proposed MRI application systems deal with pixel classification of images. While this can be justified by the high quality of MRI images which are well contrasted [18], this may not be the best approach for diagnosis of pathological cases identified by the type of tissue and shape of abnormal regions.

In this paper we propose to use the fuzzy decision tree classifier to deal with both cases of image segmentation and region shape recognition. In the first case, the pixels of input 
image are classified into predefined fuzzy regions using fuzzy decision tree (FDT) classifier. In the second case a template image is partitioned into fuzzy regions and these partitions are represented as a fuzzy decision tree (FDT). In the recognition phase, the shape of cluster of pixels at each node is matched with region properties at each node. The use of decision trees in shape classification is well known technique [10]. However, the published literature contains few comparisons between the performance of fuzzy decision tree classifier and crisp decision tree classifiers [19]. In this paper we present the experimental results conducted on sample MRI images and their error rates for different cases of sample images revealing different degrees of fuzziness.

The remainder of this paper is organized in the following manner. Section II presents the architecture of fuzzy recognition system. Section III gives the definition of the fuzzy decision tree, the induction of fuzzy decision tree and the inference of pattern classes using the fuzzy decision tree. Section IV describes the application of this system for recognition of fuzzy region in image. Then, Section $V$ presents the results of the experimental work on MRI images and quantitative comparison of CART classifier and FDT classifier.

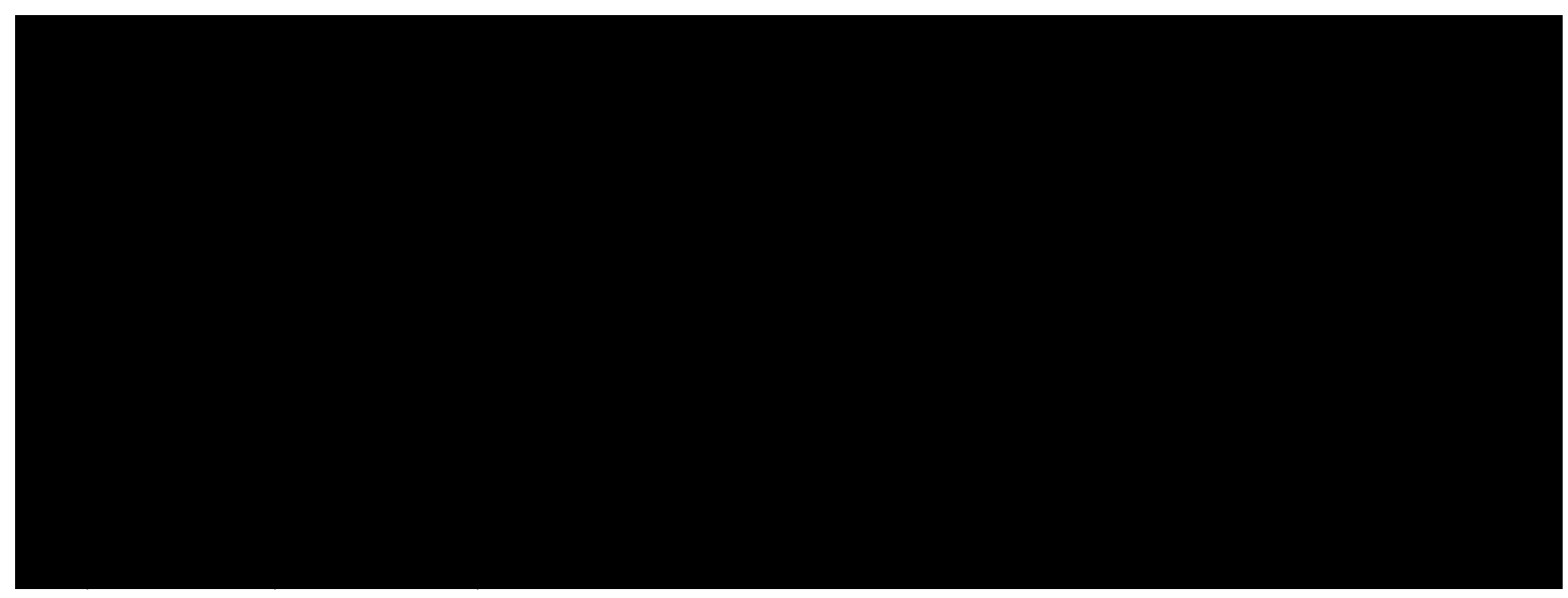

Figure 1: System Architecture

\section{Fuzzy Recognition System:}

Figure. 1 shows the proposed system architecture, which is explained as follows. Image acquisition block grabs the image and logs it into memory. The pixel features extraction block extracts intensity, texture and intensity gradient. These features are then normalized to their maximum values for further analysis.

The pixel features are stored as vectors for all image pixels.

The table of unlabeled features is entered to FCM clustering algorithm to partition these 
pixels into classes with similar features [4]. The FCM procedure is preceded by a search for optimum number of cluster. The topological region extraction block transforms the segmented region into topologically connected region. The region features extraction block measures the topological properties like area, major and minor axes, extent, solidity and convex hull. These features are then normalized before their use in classification of the region.

During the learning phase the construction of crisp decision tree from the set of labelled samples are collected from the image and labelled by the FCM algorithm. We used The CART algorithm for crisp decision tree construction then the decision tree is fuzzified by a procedure which we developed and implemented for this research work. The fuzzification procedure is similar to the published work by [6].The fuzzy decision tree algorithm extracts a fuzzy tree model of the input image. This fuzzy tree model is stored in database for later comparison with new test images.

In the recognition phase the pixel features and region features are classified by fuzzy inference algorithm base on fuzzy tree models of training sample images.

Other blocks of the system are used for the validation of the fuzzy decision tree and evaluation of the system performance.

\section{Fuzzy Decision Tree:}

\section{Definition}

A crisp decision tree is a mathematical structure for representation of set rules for classification of patterns. When a pattern is presented to the decision tree its features are examined sequentially at nodes of the tree and the pattern is directed to child node continuously until it is pushed down to a leaf of the tree which assigns the pattern to an interpretation class. A fuzzy decision tree is similar in its structure to crisp decision tree but its construction and use for classification of patterns is different. In the case of crisp decision tree, the decision to which branch of the tree the pattern should be directed depends on a crisp threshold value of the feature associated to this node. In fuzzy decision tree, the features are fuzzy and hence the decision threshold should be defined on a fuzzy set.

Formally, a fuzzy decision tree is defined [13], [16] and [17] by the 7 tuple, t,

$t=\{V, L, G, \Omega, \alpha, v\}\}$,

Where

$V$ Is the set of tree nodes,

$V=\left\{V_{1}, V_{2}, \mathrm{~L}, V_{n}\right\}$,

$L$ Is the set of tree leaves, 
$L=\left\{1_{1}, l_{2} \mathrm{~L}, l_{s}\right\}$,

$G$ Is a partial order relation on the union of the sets of nodes and leaves, $(V \cup L)$, $G\left(V_{j}\right)=\left\{V_{j} \in(V \cup L):\left(V_{j}, V_{i}\right) \in B\right\}$,

$B$ Is the set of the tree branches,

$B=\left\{\left(V_{j}, V_{i}\right)\right\}$,

$X$ Is the set of features,

$X=\left\{X^{1}, X^{2}, \mathrm{~L}, X^{n}\right\}$,

$X^{j}$ Is the mapping of the set of examples; $E$; into the set of fuzzy terms, $D^{j}$, $X^{j}: E \rightarrow D^{j}$,

$E$ Is the set of examples,

$E=\left\{e_{i} \mid e_{i}=\left(x_{i}^{1}, \ldots, x_{i}^{n}, \omega_{i}\right)\right\}$,

$D^{j}$ is the set of fuzzy terms of feature $X^{j}$,

$D^{j}=\left\{x_{p}^{j}\right\}$,

$D_{c}$ is the set of fuzzy terms for the decision variable,

$x_{p}^{j}$ is the fuzzy term (fuzzy set) $\mathrm{p}$ for the feature $X^{j}$,

$x^{j}$ is crisp value of the feature $X^{j}$,

$x^{j} \in \operatorname{dom}\left(X^{j}\right)$.

$\Omega$ is the set of interpretation classes,

$\Omega=\left\{\omega_{1}, \omega_{2}, \mathrm{~L}, \omega_{c}\right\}$,

$\alpha$ Is a surjective mapping associating to each leaf, $l_{r}$, of the tree a class $\omega_{r}$, $\alpha: L \rightarrow \Omega$,

$v$ is a surjective mapping associating to each node of the tree a feature, $X^{j}$, $v: V \rightarrow X$,

$\chi^{V}$ is the set of memberships of training examples in node $V$, $\chi^{V}=\left\{\chi_{i}^{V}\right\}$,

In this formal framework, the supervised learning problem is defined as that of searching the mappings $\alpha$, and $v$ that optimise the criterion $J(t, E)$.

In the text we will use the following additional notation due to [13] to refer to paths of the tree and children of a node.

$F^{V}$ Is the set of fuzzy restrictions on the path leading to node $V$,

$F^{V}=\left\{\left[X^{j}\right.\right.$ is $\left.\left.x_{p}^{j}\right]\right\}$

$X^{V}$ Is the set of features appearing on the path leading to the node $V$,

$X^{V}=\left\{X^{j} \mid \exists\left(\left[X^{J}\right.\right.\right.$ is $\left.\left.x_{p}^{j}\right] \in F^{V}\right\}$ 
$V \mid x_{p}^{J}$ is the child of node $V$ created by using $X^{j}$ to split $V$ and following the edge $x_{p}^{j} \in D^{j}$.

$S_{X}^{V}$ Denotes the set of $V$ 's children when created by using $X^{j}$ to split $V$

$U$ Is the universe of discourse described by a membership function, $\mu_{X}(X): U \rightarrow[0,1]$

Figure 2 below shows a sample FDT with the symbols used for it is definition.

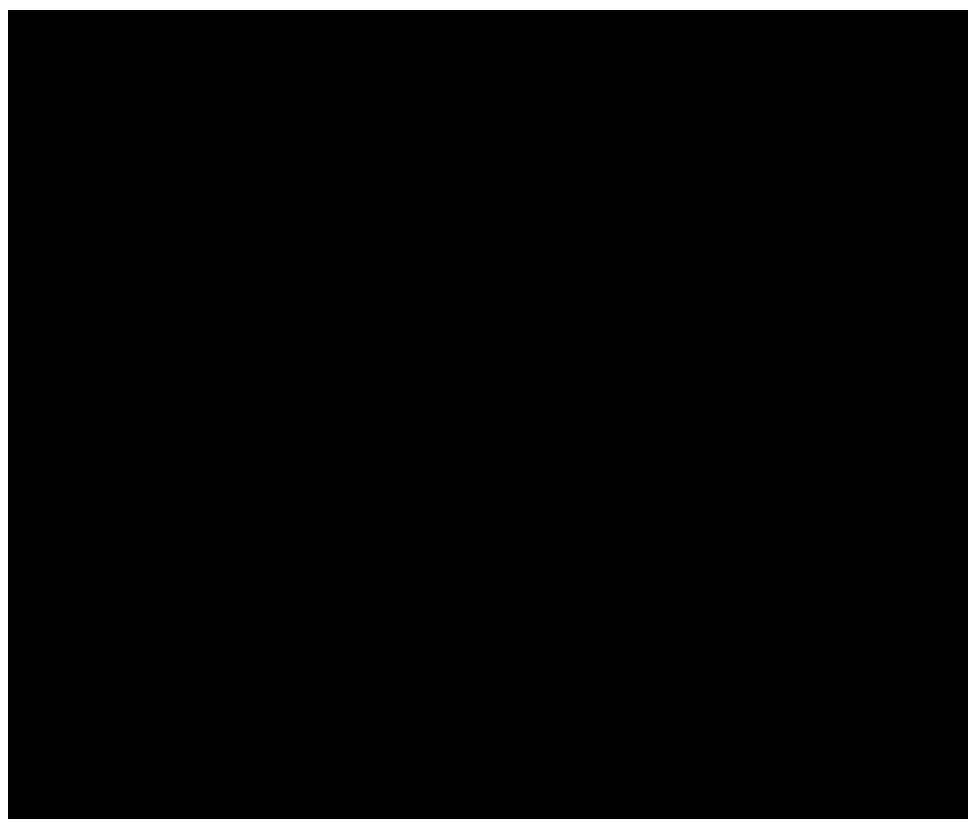

Figure 2: Fuzzy Decision Tree

\section{Induction of Fuzzy decision Tree Classifier}

A fuzzy decision tree is constructed by allowing the partial membership of a pattern in the nodes of the tree. This extends its expressive power to be a powerful functional approximator. Several methods have been proposed for induction of fuzzy decision trees [7], [19], but all of these methods require the fuzzification of pattern features prior to tree induction. Fuzzification of features is usually subjective procedure and time consuming.

The induction of fuzzy decision tree is similar in its mechanism to induction of decision trees such as ID3, CART and C4.5. The basic mechanism partitions the sample space in a data driven manner [13] and represent the partition as a tree. The partitioning process is recursive and the process stops at a leaf of the tree whenever the splitting criterion is not satisfied [21]. The FID3 uses such an approach but it requires the fuzzification of sample attributes before tree induction. In this paper we adopt the technique proposed by [6] for tree construction starting from a crisp decision tree. This approach avoids the 
need for feature fuzzification. The steps for generation of a fuzzy decision tree from set of training examples of real-valued features are as follows:

1. Generate a CART -type decision tree.

2. Prune the decision tree using predefined error rate.

3. Fuzzify the decision threshold at each tree node by specification of a pair of membership functions.

4. Optimize the shape of the membership functions.

The principle of fuzzification of decision threshold is illustrated in Figure 3.

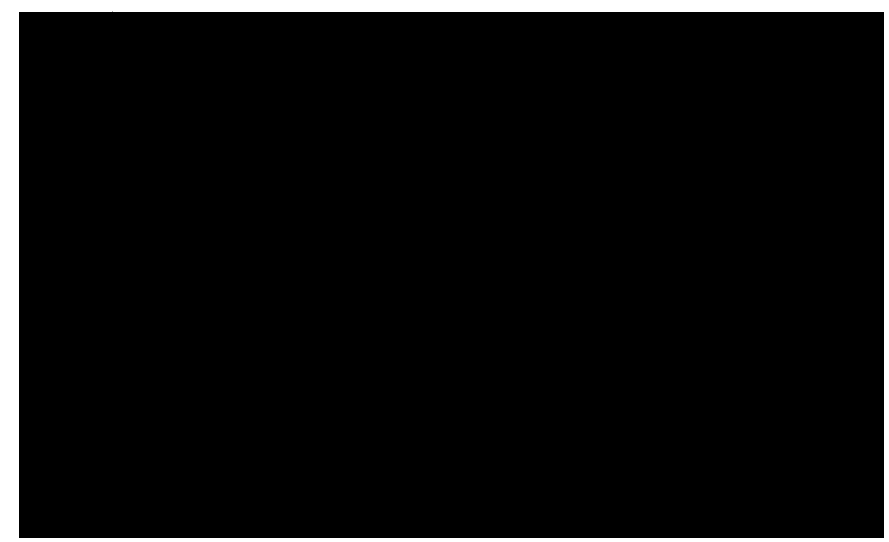

Figure 3: Fuzzification of decision threshold at tree node

A fuzzy region around any decision node is defined using a pair of complementary membership functions around a decision threshold. A specific value $x^{j}$ of feature $X^{j}$, passing through the node $V_{k}$ is a degree of membership to the acceptance region and a degree of membership to the rejection region. The example containing this feature value will be assigned to these two regions with different degrees of memberships. The domain of both membership functions, $\left[x_{l}^{k}, x_{r}^{k}\right]$ is defined by [6]

$$
\begin{aligned}
& x_{l}^{k}=x_{j 0}-n_{j} \sigma_{j} \\
& x_{r}^{k}=x_{j 0}+n_{j} \sigma_{j}
\end{aligned}
$$

\section{Where}

$x_{j 0}$ is the crisp threshold, $\mu\left(x_{j 0}\right)=0.5$,

$\sigma_{j}$ is the standard deviation of feature $X^{j}$,

$n_{j}$ is a fuzzification parameter, $n \in[0, \infty]$

The fuzzification parameters can be determined experimentally or by optimization technique such as genetic algorithm. The fuzzy c-means procedure is also a good candidate for optimal splitting of training examples at each node. In this case the result of a fuzzy c-means (FCM) procedure is the set of memberships of training samples to each of children nodes. 
We can extend the definition of a univariate decision threshold to the multivariate decision boundary. But this is out of the scope of this work.

\section{Inference in Fuzzy Decision Tree}

The fuzzy decision tree can be converted to a set of fuzzy rules [13]. Each leaf is generating one rule. The conditions leading to the leaf generate the conjunctive antecedent, and the classification of the examples of the leaf generates the consequent. An input pattern fires simultaneously all these rules to some degree. The inference of the pattern class is obtained by defuzzification of the combined membership degrees. With this understanding, the inference procedure is as follows:

1. For every leaf of the tree

- Construct the path leading to this leaf.

- Evaluate the degree of membership of the input pattern through this path of the tree.

Decide the pattern class as that associated to the leaf generating the path with maximum degree of membership.

\section{Application}

To study the system operation and control its performance parameters, we used the synthetic image shown in Figure 4. This image illustrates many qualities that are found in real-world topographic imaging of human heads. The bright elliptical shell along the exterior is analogous to a skull and the many ellipses inside are analogous to brain features or tumors.

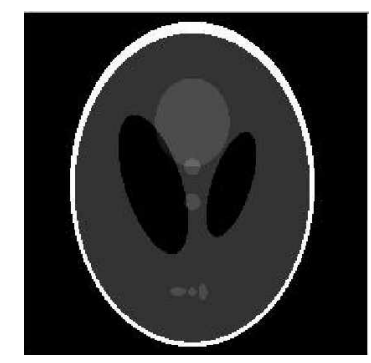

Figure 4 A synthetic topographic image of human head

After image acquisition, it's usually necessary to enhance the image quality by smoothing and contrast equalization operations. Then pixels features, including intensity, texture, gradient and position, are extracted. The FCM algorithm is used to cluster the pixels into a number of clusters based on pixels features. In FCM, the number of clusters is a key parameter [1], [2], [3], [11] and [14]. To choose the 
optimum number of clusters we apply the fuzzy clustering validity function. This measures the overall average compactness and separation of a fuzzy c-partition. A good separation validity criterion, SC, is defined as [15]:

$\mathrm{S} C=\frac{\sum_{i=1}^{c} \sum_{j=1}^{n} u_{i j}^{2}\left\|V_{i}-X_{j}\right\|^{2}}{n \min _{i, j}\left\|V_{i}-V_{j}\right\|^{2}}$

Where $\mathrm{X}_{\mathrm{j}}$ is the sample feature vector, $\mathrm{V}_{\mathrm{i}}$ is the cluster center, $\mathrm{n}$ is the number of samples, $\mathrm{c}$ is the number of clusters.

The optimum number of clusters is obtained by iteratively applying the FCM algorithm and using the separation validity criterion to stop iterations when a minimum value of separation validity is reached or the maximum allowable number of clusters is attained. Figure 5 shows the plot of separation validity against the number of clusters for the synthetic image in Figure 4. The optimum number of clusters, $c_{\text {opt }}$, found in this case is 6 , while the maximum allowable number of clusters to stop iteration was fixed to be 8 clusters. The result of fuzzy segmentation is shown in Figure 6. The FCM algorithm computes also the partition matrix of clusters which represent the degrees of memberships of image pixels to each cluster. The size and shape of each cluster depends on the degree of membership of a pixel to be assigned to this cluster. This means that for different levels of membership's degrees we obtain different shapes of clusters and consequently image regions. A level cut represents the degree of certainty that a group of pixels belong to a given cluster. The partition matrix can be used to divide the each cluster into sub clusters at different levels cuts. A plot of the degrees of memberships of pixels assigned to each cluster and represent at level-cuts is demonstrated in Figure 7. 
Figure 5 Plot of cluster separation function against number of clusters for the image in Figure 4

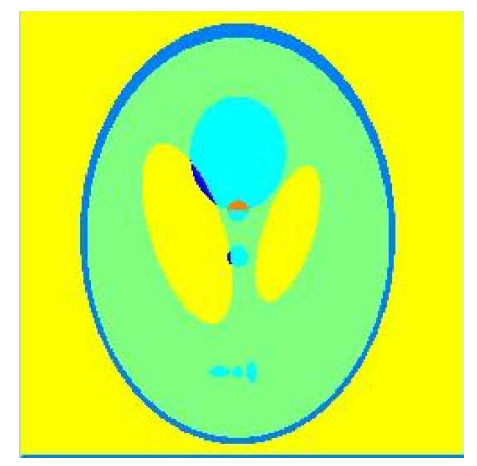

Figure 6 FCM segmented image

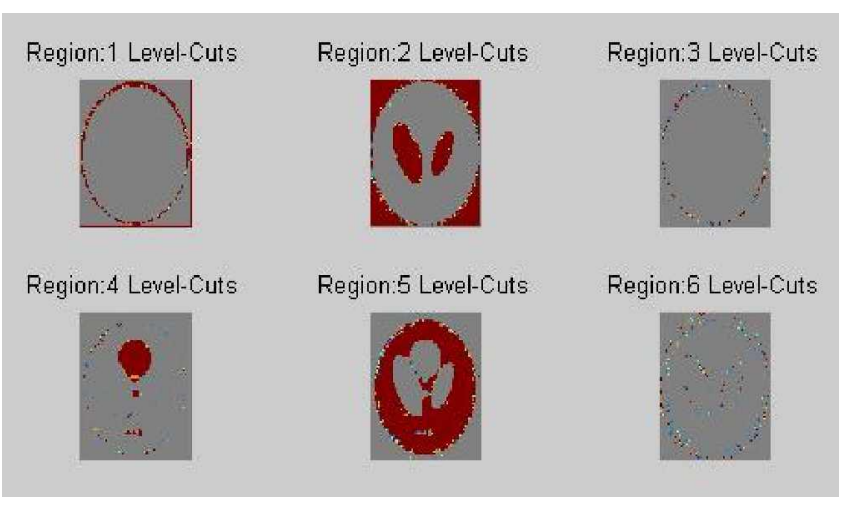

Figure 7 Levels cuts of segmented image 
The FCM segmentation procedure splits the image into regions of similar pixels features e.g. intensity in our example, but can't grantee that the pixels of the region are topologically connected. For this reason, morphological region analysis is applied to split intensity regions into eight-connected regions of pre-specified shape properties [10]. This analysis is implemented in two processing steps. At the first step, region growing algorithm is applied to extract topologically connected regions. At the second step, the filled area representation of the extracted region is analyzed and regions whose areas are blew certain threshold area are merged to their super set regions. Also, holes present in a region are identified as separate sub-regions belonging to that region.

Figure 8 below shows the result of topological regions segmentation step for the image in Figure 4. In this figure, 12 regions are identified and each topological region is painted in a different color. The convex hull of each region is also drawn in black. The convex hull will be used as a support for the membership function model of spatial fuzzy regions.

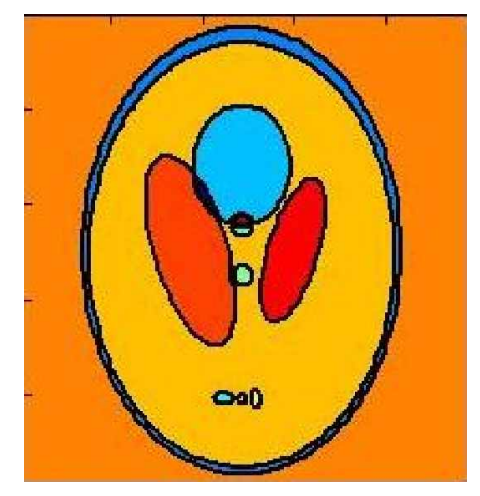

Figure 8 Topological region segmentation of the image in Figure 4

After extraction of fuzzy regions and their properties, the fuzzy decision tree can be constructed as explained in section 0 . The nodes of the fuzzy decision tree will contain two sets of information: the decision boundaries in the feature space and the features of fuzzy regions. Only decision boundaries will be used during tree construction, while features of topological regions will be used during inference on new sample image. Figure 9 shows the fuzzy decision tree of segmented image in figure 8 


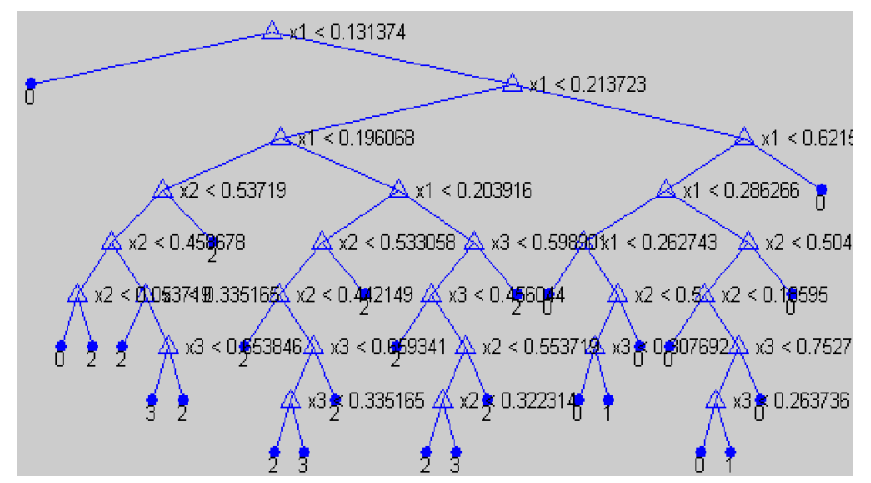

Figure 9 Fuzzy decision tree model of segmented image in Figure 8 Experimental RESULTS

The system model presented previously for learning and recognition of shapes by fuzzy decision tree classifier has been applied to sample images acquired by magnetic resonance imaging (MRI) technology. MRI has many applications in the medical domain for detecting anomalies in brain tissues or fractures in bones [5]. Figure 10 shows an example of three different images obtained by measuring three different properties in MR brain imaging. These are, from left to right: PD, T1, and T2 respectively.

The tissues of interest contained in such slices are white matter, gray matter and cerebro-spinal fluid (CSF). Although the slices presented in Figure 10 represent normal brain case, abnormal cases will show the presence of white pixel intensities inside regions of gray matter. Other cases of interest are characterized by the increase of CSF region size. The segmentation of MRI slices and detection of abnormal regions requires highly qualified experts and its time consuming process. Automatic segmentation for identification of abnormal tissues is widely used to solve this problem [5], [9] and [18]. In this paper we classify image pixels on the basis of pixel intensities and location.



Figure 10 Three slices of MRI image

The fuzzy decision tree is used for this application in either of two procedures. The first procedure aims at classification of brain tissues of a sample image into normal and abnormal tissues. The second procedure aims at identification of shape of abnormal regions in the sample image. 
In the first procedure, after construction of fuzzy decision tree a new sample image is entered to the system and pixels of the image are classified by the fuzzy decision tree into one of the leaf nodes of the fuzzy decision tree. This procedure is analogous to a fuzzy segmentation of the image. If image pixels belong to the tree model they will be classified with minimum error while if they don't belong to the tree model, they will be classified with higher error rate.

In the second procedure, the fuzzy decision tree is also used for identifying topological regions which represent normal or abnormal regions on MRI image.

Once image pixels are classified into a tree leaf, the shape properties of the aggregation of pixels at this leaf are calculated and compared with shape properties of the fuzzy region stored at this leaf.

This comparison is done according to the similarity measure between two vectors of region properties. If this similarity measure exceeds certain threshold, the classified aggregations of pixel are considered similar to the fuzzy region represented at this node else, this classified region is considered as not belonging to this tree leaf. Consequently the input image is considered as not belonging to this fuzzy tree model. This procedure is repeated at every node of the fuzzy decision tree to find matches between sub images at different levels of the fuzzy decision tree model.

To study the performance of the segmentation procedure on MRI slices we conducted experiments on a normal T1 image shown in Figure 11.

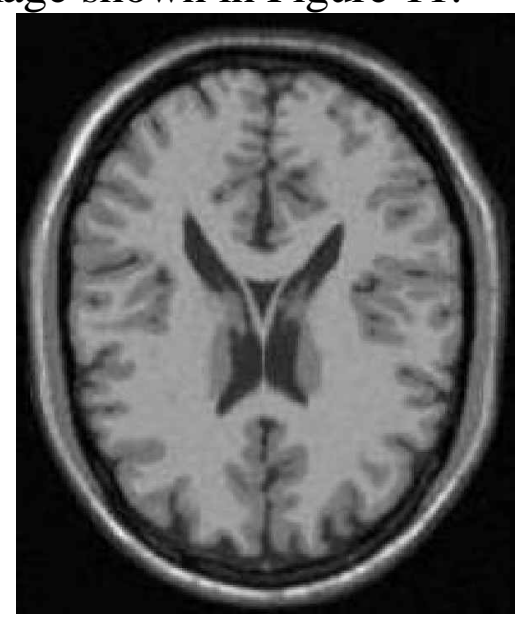

Figure 11 Normal T1 image

In the first phase, the learning procedure explained in section 2 was applied to induce the fuzzy decision tree classifier from the sample image in Figure 11. In the second phase another sample T1 image is presented to classifier to classify pixels into classes of regions stored at leaves of the tree. Sample images are generated from the original T1 image by filtering to simulate images with different degrees of blurring: low, medium, high and very high. 
The result of fuzzy segmentation is shown in Figure 12. A plot of level-cuts associated to each region demonstrated in Figure 13. Figure 14 below shows the result of topological regions segmentation step. The fuzzy decision tree for tissue segmentation is shown in Figure 15.

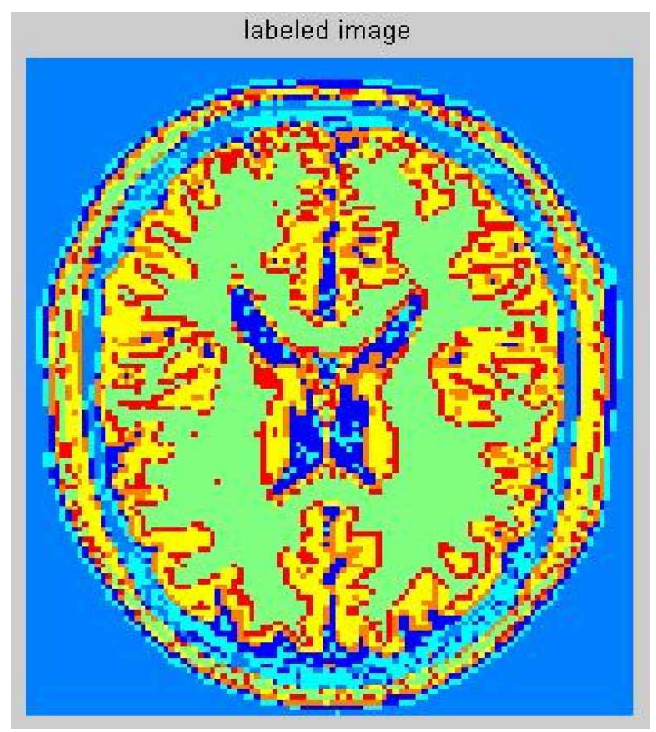

Figure 12 FCM segmentation of MRI image

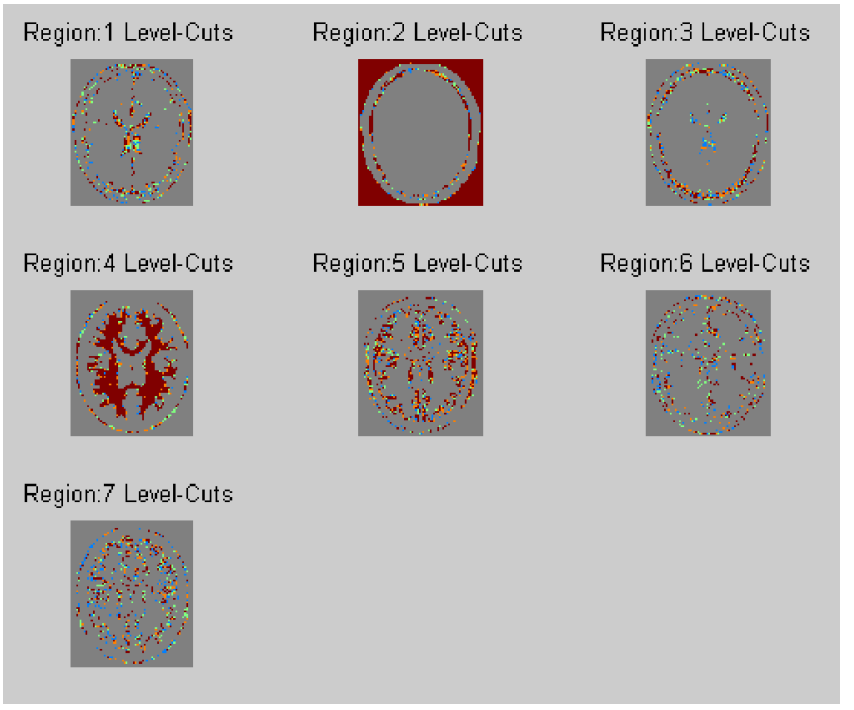

Figure 13 Levels cuts of segmented MRI image 


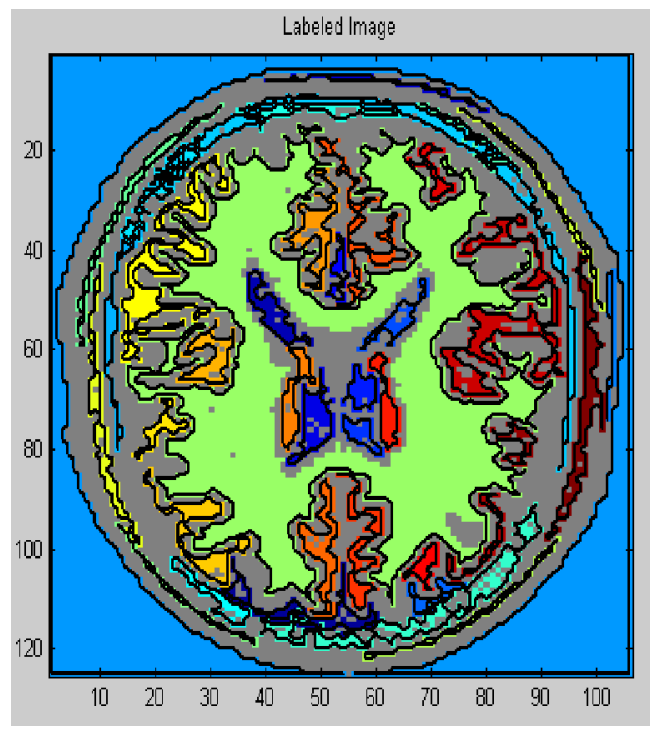

Figure 14 Connected regions of segmented MRI image

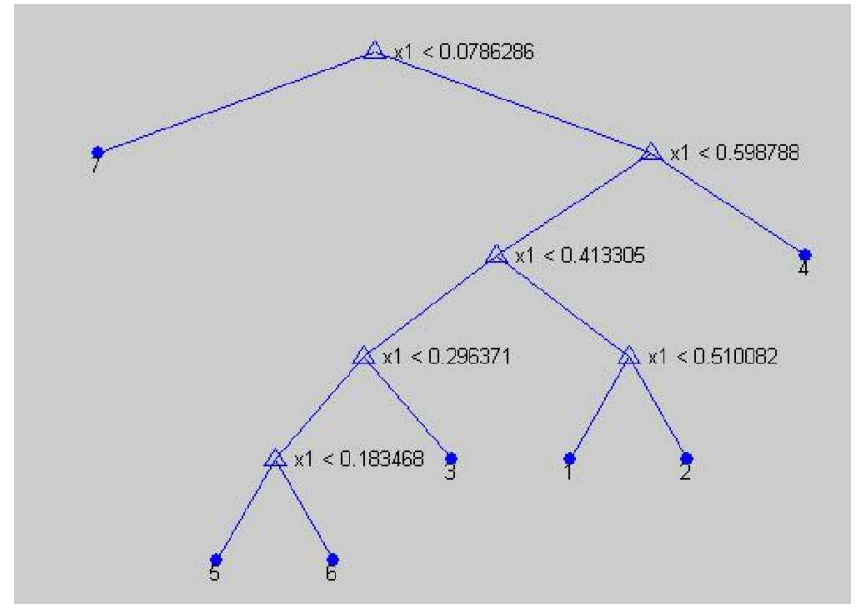

Figure 15 Fuzzy decision tree model of T1 weighted MRI image

Results of error rates of the fuzzy decision tree classifier and crisp decision tree for the synthetic image are summarized in Table 1. These results show that the error rates for both classifiers increase with the increase of degree of blur as should be expected. Only on the absence of blur the performance of CART classifier is similar to the performance of FDT classifier. In all other cases the FDT classifier is slightly better, error rate difference is 0.0001 , than the CART classifier.

Results of error rates of the fuzzy decision tree classifier Vs CART classifier for MRI T1 slice image are summarized in Table 2 . Again these results show that the error rates for both classifiers increase with the increase of degree of blur but the rate of increase is higher than in the case of synthetic image. In all cases the FDT classifier is slightly better, error rate difference is 0.001, than the CART classifier. As expected FDT classifier is more robust for classification of real world images. 
While the error rates presented here are significant, they can be reduced for the FDT classifier if the splitting membership functions at each node are optimized. Optimization technique such as genetic algorithm can be used for this optimization task.

Table 1 Error rates of the Fuzzy Decision Tree classifier Vs CART classifier for synthesized head topographic image.

\begin{tabular}{|c|c|c||}
\hline BLURING & FUZZY & CRISP \\
DEGREE & $\begin{array}{l}\text { DECISION } \\
\text { TREE } \\
\text { CLASSIFIER } \\
\text { TREE } \\
\text { CLASSIFIER }\end{array}$ \\
\hline \hline Non & 0.00081736 & 0.00081736 \\
\hline Low & 0.014486 & 0.014463 \\
\hline Medium & 0.040119 & 0.040096 \\
\hline High & 0.065593 & 0.065616 \\
\hline Very High & 0.073495 & 0.073563 \\
\hline
\end{tabular}

Table 2 Error rates of the Fuzzy Decision Tree classifier Vs CART classifier for MRI T1 slice.

\begin{tabular}{||c|c|c||}
\hline BLURING & $\begin{array}{l}\text { FUZZY } \\
\text { DEGREE } \\
\text { TREISION } \\
\text { CLASSIFIER }\end{array}$ & $\begin{array}{l}\text { CRISP } \\
\text { DECISION } \\
\text { TREE } \\
\text { CLASSIFIER }\end{array}$ \\
\hline \hline Very Low & 0.019928 & 0.021739 \\
\hline Low & 0.2126 & 0.21321 \\
\hline Medium & 0.24204 & 0.24226 \\
\hline High & 0.26211 & 0.26347 \\
\hline Very High & 0.28453 & 0.28506 \\
\hline
\end{tabular}

Conclusion

In this paper we presented a framework for image segmentation into fuzzy regions and their subsequent recognition using a fuzzy decision tree classifier.

The system architecture has been implemented and tested in MATLAB environment. The system response time for learning and recognition phase is acceptable for real time applications. We implemented the algorithm of fuzzy decision tree construction by node fuzzification.

To compare the classification error rate of the fuzzy decision tree classifier with CART classifier, we tested both classifiers through samples of synthetic images and MRI images with different degrees of blur. 
In test cases of sample synthetic image, only on the absence of blur the CART classifier has similar performance to the FDT classifier. In all other cases the FDT classifier is slightly better than the CART classifier by error rate difference of 0.0001 .

In all test cases of sample MRI images, the FDT classifier is slightly better than the CART classifier by error rate difference of 0.001 . This result confirms the robustness of FDT classifier for classification of real world images.

\section{References}

[1] Andrea Baraldi and Palma Blonda, Member, IEEE "A survey of fuzzy clustering algorithms for pattern recognition -part I" IEEE transactions on systems, man, and cybernetics — part B: cybernetics, vol. 29, NO. 6, December 1999.

[2] Andrea Baraldi and Palma Blonda, Member, IEEE "A survey of fuzzy clustering algorithms for pattern recognition-part II" IEEE transactions on systems, man, and cybernetics — part B: cybernetics, vol. 29, NO. 6, December 1999.

[3] James C. Besdek, Robert Ehrlich William Full " FCM: The fuzzy c-means clustering algorithm " Computers \& Geosciences vol. 10, no. 2-3, pp. 191-203, 1984.

[4] I. GATH A D A. B. GEVA, "Unsupervised optimal fuzzy clustering" IEEE transactions on pattern analysis and machine intelligence. vol. I I . no. 7. July 1989.

[5] Sarah E. Crane and Lawrence O. Hall "Learning to identify fuzzy regions in magnetic resonance images" Fuzzy Information Processing Society, 1999. NAFIPS. 18th International Conference of the North American, 10-12 June 1999.

[6] Keeley Crockett,Zuhair Bandar, David Mclean, James O'Shea "On constructing a fuzzy inference framework using crisp decision trees" The intelligent system group, department of computing, Manchester Metropolitan university July 2006.

[7] Ming Dong, Student Member, IEEE, and Ravi Kothari, Senior Member, IEEE, "Look-ahead based fuzzy decision tree induction" IEEE transactions on fuzzy systems, vol. 9, no. 3, June 2001.

[8] Richard O. Duda, Peter E. Hart and David G. Stork, "Pattern Classification", (2nd ed.), Wiley Interscience, 2001.

[9] Mohsen Ghazel, Anthony Traboulsee, Rabab K. Ward," Semi-Automated Segmentation of Multiple Sclerosis Lesions in Brain MRI using Texture Analysis ",2006 IEEE International.

[10] Rafael C. Gonzalez, Richard E. Woods, "Digital image processing", Second Edition, Prentice Hall,2002.

[11] Reginald E. Hammah and John H. Curran," Validity measures for the fuzzy cluster analysis of orientations" IEEE transactions on pattern analysis and machine intelligence, vol. 22, no. 12, December 2000.

[12] Adel Hlaoui,HAOJUN SUN,SHENGRUI WANG "Image retrieval using fuzzy segmentation and a graph matching technique" December 2005. 
[13] Cezary Z. Janikow, "Fuzzy Decision Tree: Issues and Methods" IEEE transactions on systems, man , and cybernetics, vol. 28, no. 1 February 1998.

[14] Kuo-Lung Wu a, Miin-Shen Yang "A cluster validity index for fuzzy clustering" K.-L. Wu, M.-S. Yang / pattern recognition letters 26 (2005) 1275-1291.

[15] Xunali Lisa Xie and Gerardo Beni "A validity measure for fuzzy clustering" IEEE transactions on pattern analysis and machine intelligence, vol. 13, no.8, August 1991.

[16] M.Y. Mahmoud (El Nahas) \& J.P. Bonnefoy et al: "Un algorithme séquentiel autoadaptatif réalisant un apprentissage automatique avec ou sans professeur", Expersys-92, Paris, october 1992.

[17] Ferenc Peter Pach, Janos Abonyi, Sandor Nemeth and Peter Arva, "Supervised clustering and fuzzy decision tree induction

for the identification of compact classifiers", http://www.bmf.hu/conferences/mtn/abonyi.pdf.

[18] Selvathi. D \& Arulmurgan, Sivakasi-626005, Tamil Nadu, India, Devaki MRI \& CT Scans, Madurai - 620015 Tamil Nadu, India, "MRI Image Segmentation Using Unsupervised Clustering Techniques", Proceedings of the Sixth International Conference on Computational Intelligence and Multimedia Applications (ICCIMA'05), 2005 IEEE.

[19] Alberto SuaÂ rez and James F. Lutsko, "Globally optimal fuzzy decision trees for classification and regression" IEEE transactions on pattern analysis and machine intelligence, vol. 21, no. 12, December 1999.

[20] Sinisa Todorovic, and Michael C. Nechyba, "Dynamic Trees for Unsupervised Segmentation and Matching of Image Regions", IEEE transactions on pattern analysis and machine intelligence, vol. 27, no.11, Novemeber 2005.

[21] Xiaomeng Wang, Christian Borgelt "Information measures in fuzzy decision trees" department of computer Science University of Magdeburg 39106 Magdeburg, Germany.

[22] Laurent Wendling,Jacky Desachy,Alain Paries, "Pattern recognition by splitting images into trees of fuzzy regions", December 1996. 